\title{
LA FORMOLIZACIÓN DE ELTIEMPO.COM FRENTE A LA VIVIFICACIÓN DE ELESPECTADOR.COM : LOS ENLACES HIPERTEXTUALES EN LOS CONTENIDOS SOBRE EL PRESIDENTE SANTOS $(2010)^{1}$
}

\author{
THE PRESERVATION OF ELTIEMPO.COM IN FRONT OF THE \\ VIVIFICATION OF ELESPECTADOR.COM: HYPERTEXT LINKS IN THE \\ CONTENTS ABOUT THE PRESIDENT SANTOS (2010)
}

\author{
Por: \\ Daniel Barredo Ibáñez \\ Doctor en Periodismo \\ Universidad de Málaga \\ danielbarredo@aol.com
}

Resumen: Los enlaces hipertextuales son una de las principales estrategias utilizadas para interconectar los contenidos en los cibermedios. No solo son estrategias retóricas propias de la narrativa digital, sino que su empleo configura un nuevo tipo de lenguaje: el lenguaje hipertextual. En este estudio, mediante el análisis de una información simbólicamente representativa del quehacer ciberperiodístico colombiano (las noticias relativas al presidente Juan Manuel Santos), se clasifican los niveles de implementación de los enlaces de casi tres mil contenidos publicados en eltiempo.com y elespectador.com en 2010. Como conclusiones generales se han avistado dos preferencias en el almacenaje de los contenidos: la formolización, un proceso con el que se preserva el texto, aunque se eliminan esas partes susceptibles de causar errores, como los enlaces. Y la vivificación, la intensificación de la experiencia del usuario mediante la instalación de máquinas agregadoras automáticas. Ambos son procesos hegemónicos, en tanto que un cibermedio ha de optar por uno u otro.

Palabras Clave: eltiempo.com, elespectador.com, enlaces hipertextuales, presidente Santos. 


\begin{abstract}
Hypertext links are one of the main strategies used to interconnect contents in the digital media. They are not just rhetoric strategies inherent to the digital narrative, but also its implementation configures a new kind of language: the hypertext language. In this article, with the analysis of a symbolic information representative of the digital Colombian journalism (the news about the President Juan Manuel Santos), we have classified the levels of implementation of links of nearly three thousand contents published in eltiempo.com and elespectador.com in 2010. As general conclusions we have observed two preferences to store the digital contents: the preservation, a process which preserves the text, but without those parts which can cause errors, as for example the links. And the vivification, which is the intensification of the experience of the users with the installation of automatic aggregator machines. Both processes are hegemonic, because the digital media have to choose one or the other.
\end{abstract}

Keywords: eltiempo.com, elespectador.com, hypertext links, president Santos

\title{
INTRODUCCIÓN
}

Los enlaces hipertextuales son una de las principales estrategias utilizadas para interconectar los contenidos en los cibermedios, como aseguran Díaz Noci, Codina, da Fonseca et al. (2009) o Armentia, Caminos, Elexgaray et al. (2000). Un enlace, como su nombre indica, es un "nodo" (Waniek, 2012, p.143) que vincula un elemento (por ejemplo una palabra, línea de tex to o imagen) con otro elemento (otra noticia o recurso). Algunos autores, como por ejemplo Larrondo (2010), han relacionado el empleo del hipertexto con el de los recursos estilísticos en la literatura. O sea: no solo son estrategias retóricas propias de la narrativa digital, sino que su empleo configura un nuevo tipo de lenguaje: el lenguaje hipertextual. Ese lenguaje apuntala un discurso multipolarizado, en tanto que se fragmenta la linealidad del texto escrito. Aunque el nacimiento del hipertexto se asocia habitualmente al desarrollo de la informática ${ }^{2}$, Larrondo (2009, p. 71) explica su génesis con ideas como la "intertextualidad" barthesiana, el "dialogismo" bajtiniano y otras corrientes hermenéuticas aparecidas durante el siglo XX. El texto hipertextual o multipolarizado determina asimismo la aparición de un nuevo tipo de receptor: un receptor que abandona la actitud pasiva y se transforma en un activo creador de significados, ya que mediante el hipertexto los sentidos del contenido se amplían a priori indefinidamente. A medida que el usuario del cibermedio interactúa con la multipolaridad hipertextual, se generan nuevas perspectivas y sentidos, las cuales multiplican cuantitativa y cualitativamente un contenido.

En Colombia la convergencia digital se inició a finales de los años noventa, como explican Fondevila \& Segura (2012a, p. 81), pero fue en 2003 cuando los principales diarios consolidaron sus cabeceras ciberperiodísticas (Fondevila y Segura, 2012b). Es esta una fecha tardía en comparación con otros países; en EEUU, por ejemplo, el San José Mercury News apareció en digital en 1994 (en Rubio \& Blanco 2010, p. 273), mientras que en España la mayor parte de los ciberdiarios emergieron a mediados de la década de los noventa, como menciona Pérez Marco (2003). 
Quizá debido a esta tardía adaptación a las rutinas digitales, la concentración de enlaces hipertextuales en el ciberperiodismo colombiano ha sido descrita en análisis precedentes como de baja intensidad (en Llano, 2005; o en Fondevila \& Segura, 2012a). Las siguientes páginas se han fijado como objetivos generales:

1. Catalogar los enlaces hipertextuales de las informaciones relativas al presidente Juan Manuel Santos publicadas durante los primeros cinco meses de su mandato (2010).

2. Confrontar el tipo de estrategias hipertextuales gestionadas por dos de las principales tendencias periodísticas colombianas: eltiempo.com y elespectador.com.

Son objetivos que pretenden aportar algunas claves para entender la implementación del hipertexto alrededor de una información referencial -como es la relativa a la presidencia de la República de Colombia-, dentro de una línea de estudio -la hipertextualidad en los cibermedios colombianos- con una escasa tradición investigadora.

\section{METODOLOGÍA}

\section{Análisis de contenido: la ficha de análisis}

La metodología escogida ha sido el análisis de contenido, uno de los instrumentos más utilizados en los estudios en Comunicación, como comenta Neuendorf (2002). Mediante el análisis de contenido el investigador busca cuantificar determinadas características de un objeto determinado (Cea D'Ancona, 1996). Se ha empleado una ficha de análisis, cuya eficacia ha sido comprobada en algunos trabajos previos (Barredo y Oller, 2012a; 2012 b; Barredo, 2013). En total se han examinado 24 variables, las cuales pueden ser explicadas según la siguiente clasificación, determinada por las fases o grupos de variables y sus objetivos esenciales ${ }^{3}$ :

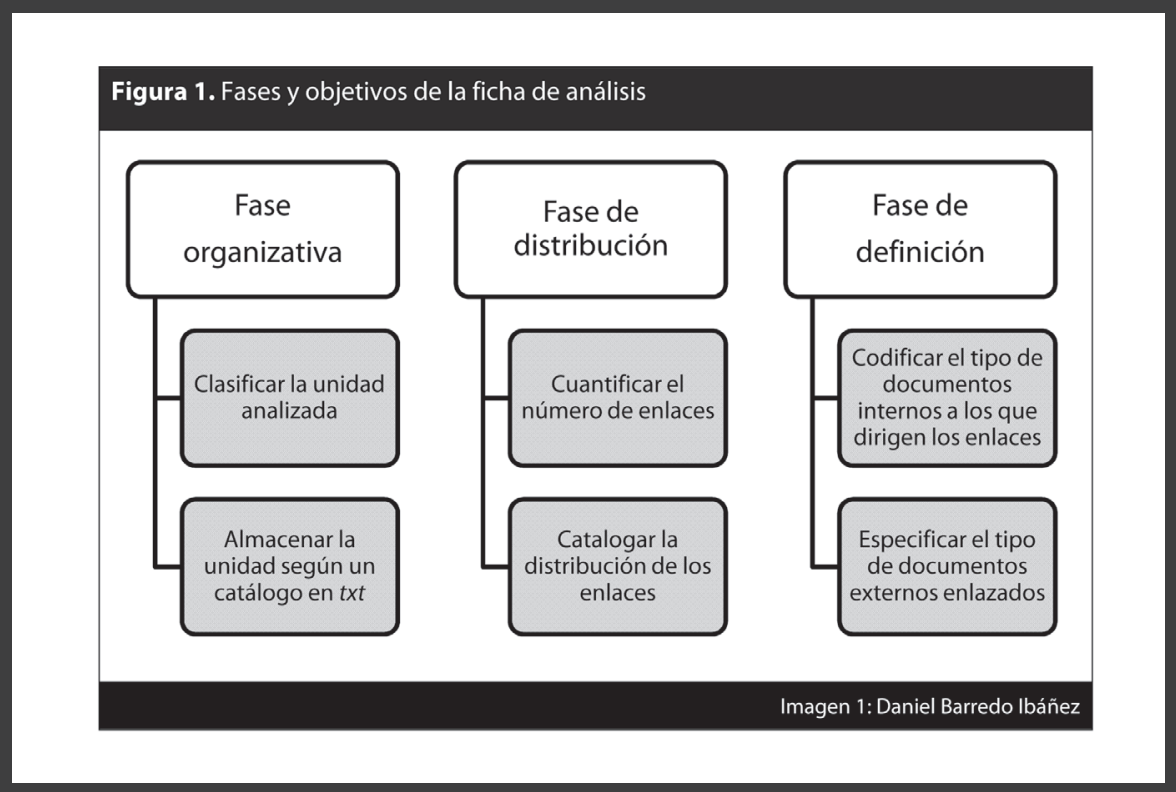


Como muestra la imagen 1, la ficha avanza gradualmente desde momentos que examinan cuestiones perimetrales de la información, hacia una mayor profundidad analítica.

\section{La elección de eltiempo.com y elespectador.com}

De entre los muchos cibermedios colombianos, se han escogido únicamente dos porque el objetivo de este análisis era examinar la inclusión y la tipología de los enlaces hipertextuales desde un punto de vista transversal. En ese sentido se ha analizado eltiempo. com porque es el primer diario digital de Colombia y se encuentra en el puesto 12 entre las páginas más visitadas del país ${ }^{4}$. Elespectador.com, por su parte, es el segundo diario digital de tirada nacional y la página número 17 entre las más visitadas de Colombia ${ }^{5}$.

\section{Fecha de búsqueda}

Se han examinado todos los contenidos alusivos al mandatario colombiano entre el 7/08/2010 y el 31/12/2010 debido a:

\section{a) Factores contextuales}

a.1) Se ha analizado un bloque de contenidos ajustado a una fecha temporal, dado el elevado dinamismo del diseño de los cibermedios. El cotejo de fechas escalonadas, por ejemplo, podría producir resultados diferentes dentro de una misma cabecera.

a.2.) Este análisis se inserta dentro de una investigación de mayor calado, basada en un procedimiento llamado análisis de contenido informatizado, el cual necesita evaluar un bloque informativo, lo que impide la realización de un muestreo probabilístico.

a.3.) La cobertura de la información presidencial puede ser indicativa del máximo grado de desarrollo técnico de unos ciberdiarios de orientación nacional, ya que a diferencia de otros apartados (como los espacios dedicados a la información local o cultural), es en la información presidencial donde suele destinarse un mayor número de profesionales y de recursos.

\section{b) Factores históricos}

b.1.) El 7 de agosto de 2010 señala el inicio de la designación de Juan Manuel Santos como jefe de Estado de Colombia.

b.2.) El 31 de diciembre de 2010 marca el final de sus primeros cinco meses de mandato. 


\section{Hemerotecas digitales}

Las unidades se han descargado desde las hemerotecas digitales de ambas cabeceras. Estas hemerotecas son accesibles a través de unas máquinas de búsqueda situadas en sus portadas. En las capturas siguientes, para facilitar su localización, se han rotulado con sendos cuadrados rojos dichas máquinas de búsqueda:

\section{Figura 2. Máquinas de búsqueda de contenidos en las portadas (20/04/2013)}

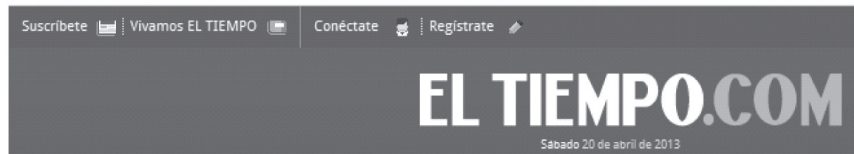

Portada I Opinión I Politica I Justicia I Bogotá IDeportes I Entretenimiento IReportajes I Tecnologia I Clasificados I Secciones

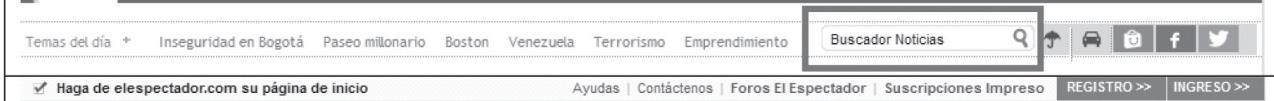

$\checkmark$ Haga de elespectador.com su página de inicio Ayudas | Contáctenos | Foros El Espectador | Suscripciones Impreso ELESPECTADOR·COM INICIO NOTICIAS $\checkmark$ OPIIIÓN ECONOMIA $\checkmark$ DEPORTES $\checkmark$ CULTURA ENTRETEMIMIENTO $\checkmark$ VIVIR $\checkmark$ TECMOLOGIA BLOGS

Una vez tecleado el comando de búsqueda (Juan Manuel Santos), ambas hemerotecas permiten clasificar los contenidos gracias a multitud de variables cuantitativas (fechas, secciones) y cualitativas (relevancia). Todos los contenidos examinados han sido descargados y archivados tanto en un catálogo en Excel, como en unas carpetas contenedoras dentro de ficheros de texto sin enriquecer ( $t x t)$.

\section{Exclusión de los enlaces}

Con el fin de centrar y estabilizar el análisis, se han efectuado algunas exclusiones. En primer lugar, se han descartado los enlaces agregados automáticamente (como los enlaces a contenidos futuros) o que aportan muy poca orientación sobre la escritura hipertextual (como los enlaces que remiten a galerías contenedoras). En las capturas siguientes mostramos dos ejemplos de esos enlaces excluidos: la galería que acompaña a la información (con fecha de 12 de noviembre de 2010), conduce a contenidos publicados el 30 de abril de 2013. Por su parte, el enlace tag a Fondelibertad remite al usuario a una galería de contenidos protagonizados por esta entidad ${ }^{6}$ : 
Figura 3. Capturas de enlaces agregados automáticamente o hacia galerías contenedoras (30/04/2013)

\section{Fondelibertad, intervenido}

Por escándalo de presunta corrupción en la entidad.

Por: Elespectador.con

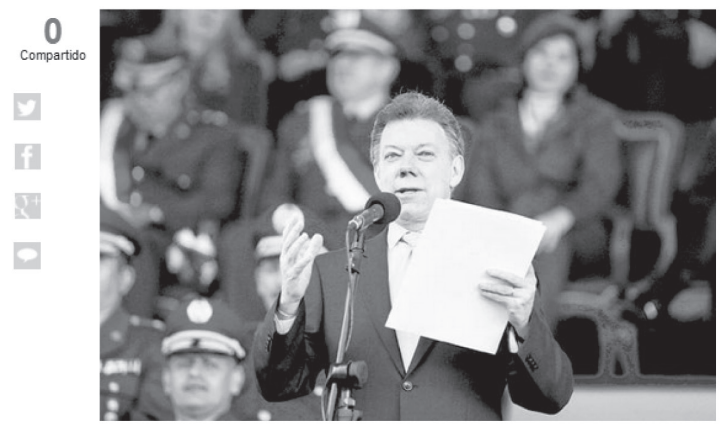

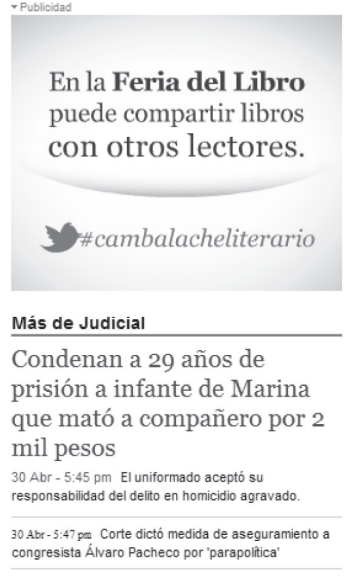

congresista Álvaro Pacheco por 'parapolitica'

Con todo, serán las autoridades las que determinen y les pongan nombres y apellidos a los responsables del episodio de Fondelibertad.

Por: Elespectador.com

Tags

Fondelibertad

Ambos tipos de enlaces explicados introducen sensaciones de lejanía con respecto de la evaluación narrativa que se ha fijado este artículo y, por lo tanto, han sido excluidos del recuento. También durante la fase de análisis se han descartado de la evaluación las unidades específicamente audiovisuales (audionoticias, fotonoticias, videonoticias), porque durante el trabajo de campo observamos que ELTIEMPO.com tiende a eliminarlas de su archivo: buscábamos, ante todo, un análisis transversal equilibrado entre ambas cabeceras.

\section{RESULTADOS}

\section{Muestra empleada}

En total se han examinado 2571 unidades, conformadas por 1.300 .999 palabras, distribuidas de las formas siguientes:

\begin{tabular}{|c|c|c|}
\hline \multicolumn{2}{|c|}{ Tabla 1. Unidades analizadas sobre el presidente Juan Manuel Santos (2010) } \\
\hline ELTIEMPO.com & ELESPECTADOR.com \\
\hline Unidades & 1178 & 1393 \\
\hline Palabras & 571008 & 729991 \\
\hline Media de palabras por unidad & 484,72 & 524,04 \\
\hline & & \multicolumn{2}{c|}{ Fuente: Daniel Barredo lbáñez }
\end{tabular}


Según la tabla anterior, se han analizado casi doscientas unidades más en ELESPECTADOR. com que en ELTIEMPO.com, una diferencia relacionada con un mayor interés informativo del primero de los ciberdiarios citados. La media de palabras por unidad, también más abultada en ELESPECTADOR.com, señala una cobertura más compleja desde el punto de vista informativo.

\section{Inclusión de enlaces}

El porcentaje de inclusión de enlaces, como comenta Madrid López (2010), se fija en función de determinadas estrategias compositivas de cada ciberdiario. Esa flexibilidad augura una dispersión que también ha sido corroborada en nuestro trabajo:

\section{Tabla 2. Enlaces en las alusiones al presidente Juan Manuel Santos (2010)}

\begin{tabular}{|c|c|c|c|c|}
\hline & \multicolumn{2}{|c|}{ ELTIEMPO.com } & \multicolumn{2}{c|}{ ELESPECTADOR.com } \\
\hline No & Veces & $\%$ & Veces & $\%$ \\
\hline 0 & 1105 & 93,8 & 846 & 60,7 \\
\hline $1-2$ & 59 & 5 & 360 & 25,8 \\
\hline $3-4$ & 9 & 0,8 & 138 & 9,9 \\
\hline $5-6$ & 2 & 0,2 & 37 & 2,7 \\
\hline $7-8$ & 1 & 0,1 & 8 & 0,6 \\
\hline $9-10$ & 1 & 0,1 & 3 & 0,2 \\
\hline 11 o más & 1 & 0,1 & 1 & 100 \\
\hline TOTAL & 1178 & 100 & 1393 & Fuente: Daniel Barredo Ibáñez \\
\hline
\end{tabular}

Globalmente, ELESPECTADOR.com tendía a incluir un mayor número de enlaces, con una media de 0,39 enlaces por unidad, en tanto que ELTIEMPO.com agregaba una media de 0,06 enlaces por unidad. En el caso de ELESPECTADOR.com hemos obtenido un porcentaje similar al de Fondevila y Segura (2012b), ya que estos investigadores aseguraban haber coleccionado una media de 0,25 enlaces por unidad ${ }^{7}$. En ELTIEMPO.com, en cambio, hemos detectado divergencias con respecto del estudio mencionado ${ }^{8}$; este hecho podría venir motivado por un cambio en las estrategias de redacción hipertextual de la cabecera, ya que el estudio mencionado emplea una muestra conformada por contenidos publicados en 2011, en tanto que para este artículo se han analizado noticias publicadas en 2010. 
Según la tabla anterior, la franja de máxima incidencia de ELESPECTADOR.com se situaba en un estrato intermedio, en la horquilla de 1 a 6 enlaces:

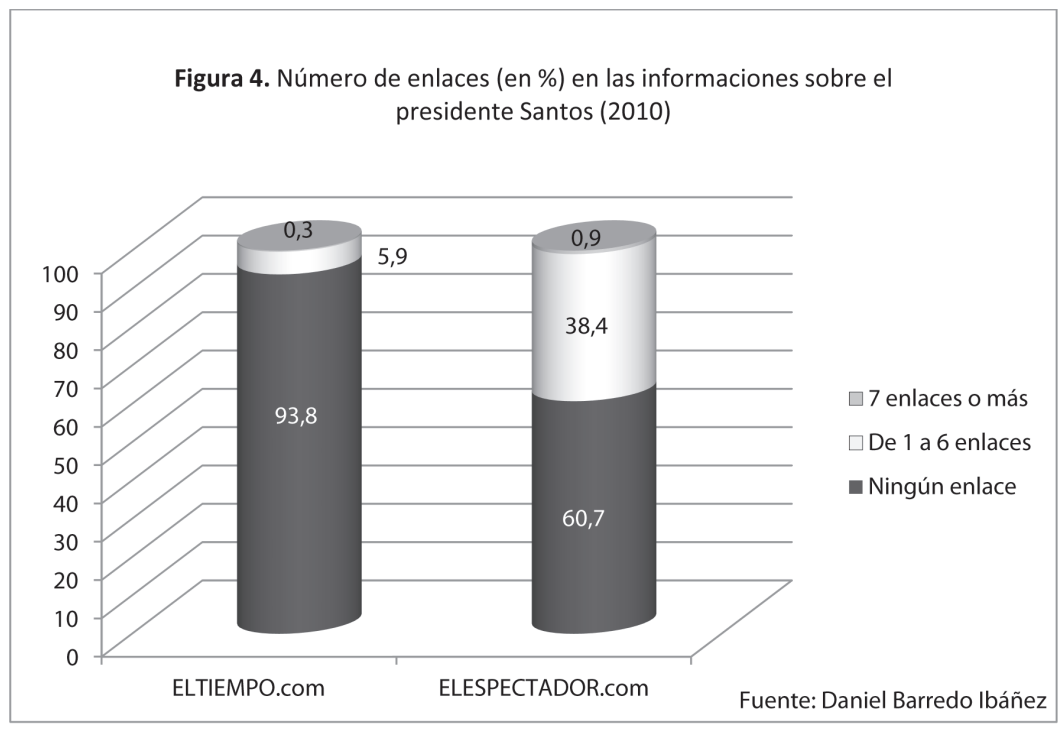

ELTIEMPO.com, en cambio, al almacenar sus contenidos tendía a eliminar los enlaces hipertextuales; el 93,8\% (n=1105) de las unidades catalogadas carecían de estas estrategias narrativas propias del lenguaje digital.

La situación zonal de los enlaces en estos dos ciberdiarios colombianos dependía asimismo de sus preferencias internas, de la coherencia del diseño, y de otros aspectos relacionados con la usabilidad de la información. En todas las áreas estructurales la incidencia de hipertexto en ELESPECTADOR.com superaba a ELTIEMPO.com, porque su porcentaje de inclusión de enlaces era -como se ha explicado- mayor:

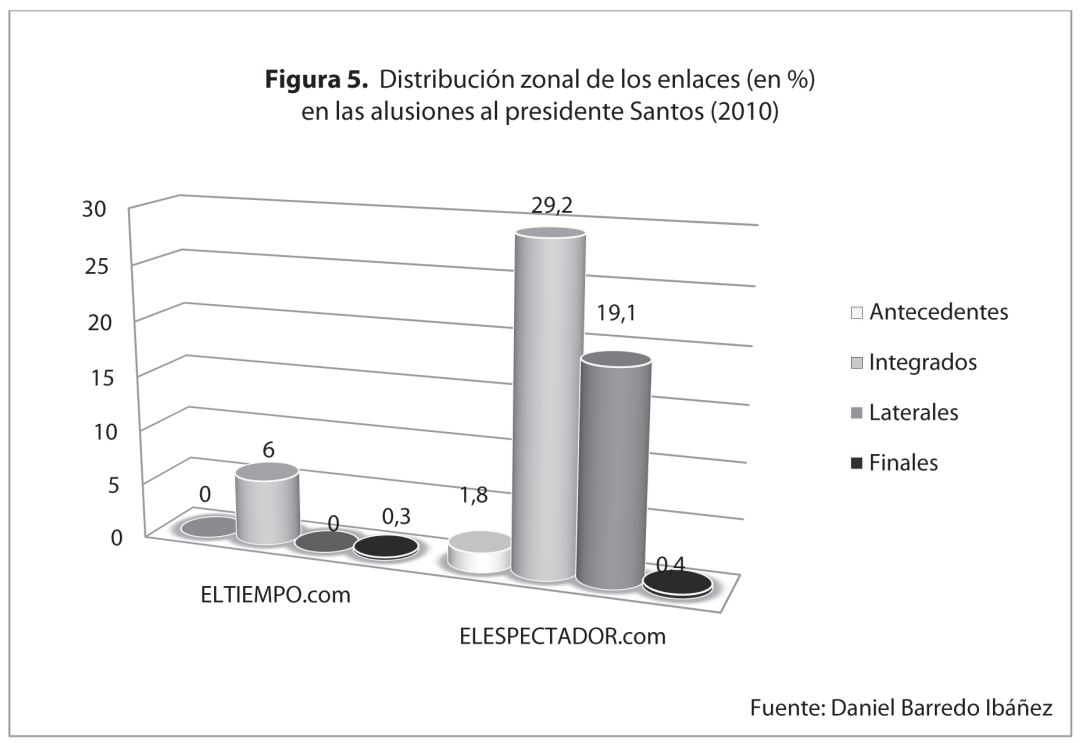


Es este un reflejo del tipo de almacenaje que estructuraba cada uno de estos ciberdiarios: ELTIEMPO.com reducía drásticamente la inclusión del hipertexto, mientras que ELESPECTADOR.com mantenía unos niveles medios de implementación. En ambos casos el grupo más abultado de enlaces se situó dentro del texto, es decir, el hipertexto aparecía integrado dentro de los otros recursos expresivos de la información.

\section{Tipología hipertextual}

Tanto en ELTIEMPO.com como en ELESPECTADOR.com los enlaces más numerosos remitían a contenidos alojados dentro de la propia cabecera; es esta una característica que ya se ha avistado en un trabajo paralelo a este estudio (Barredo, 2013). Los cibermedios, en general, son renuentes a enlazar archivos situados fuera de sus portales, principalmente por problemas como la posible infracción de los derechos de autor o la cuestión de la responsabilidad legal (en Deuze, 2001).

Dentro de los enlaces internos ${ }^{9}$, el porcentaje global de incidencia de ELESPECTADOR. com multiplicaba casi por ocho veces al de ELTIEMPO.com:

\begin{tabular}{|c|c|c|c|c|}
\hline \multirow[b]{2}{*}{ Tipos } & \multicolumn{2}{|c|}{ ELTIEMPO.com } & \multicolumn{2}{|c|}{ ELESPECTADOR.com } \\
\hline & Veces & $\%$ & Veces & $\%$ \\
\hline Archivo futuro & 0 & 0 & 0 & 0 \\
\hline Archivo pasado & 40 & 3,4 & 65 & 4,7 \\
\hline Blog & 0 & 0 & 0 & 0 \\
\hline Fichero de audio & 1 & 0,1 & 9 & 0,6 \\
\hline Fichero de vídeo & 0 & 0 & 47 & 3,4 \\
\hline Fotografía & 2 & 0,2 & 13 & 0,9 \\
\hline Infografía & 3 & 0,3 & 243 & 17,4 \\
\hline Noticia del día & 6 & 0,5 & 211 & 15,1 \\
\hline Otra noticia & 29 & 2,5 & 158 & 11,3 \\
\hline TOTAL & 81 & $7 \%$ & 746 & $53,4 \%$ \\
\hline
\end{tabular}

Cada una de las cabeceras identificaba unas tipologías hipertextuales de referencia. ELTIEMPO.com se decantaba fundamentalmente por los enlaces a contenidos de archivo pasado, y a noticias publicadas en los días inmediatamente anteriores o posteriores. ELESPECTADOR.com, por su parte, apostaba por las infografías y las noticias publicadas el mismo día o en los días inmediatamente anteriores o posteriores a los días de la información. 
Los niveles de enlaces externos ${ }^{10}$ se asemejaban en estas dos tendencias del ciberperiodismo colombiano: tanto ELTIEMPO.com como ELESPECTADOR.com tendían a enlazar, sobre todo, a fuentes externas al contenido tales como fuentes institucionales, corporaciones académicas o empresariales. Son estos los enlaces "recomendados" que mencionan Fondevila y Segura (2012a, p. 82), porque adensan la información al vincularla con documentos referenciales.

\begin{tabular}{|c|c|c|c|c|}
\hline \multirow[b]{2}{*}{ Tipos } & \multicolumn{2}{|c|}{ ELTIEMPO.com } & \multicolumn{2}{|c|}{ ELESPECTADOR.com } \\
\hline & Veces & $\%$ & Veces & $\%$ \\
\hline Documentos originales & 1 & 0,1 & 12 & 0,9 \\
\hline Fuentes externas & 11 & 0,9 & 14 & 1 \\
\hline Medios del grupo & 2 & 0,2 & 0 & 0 \\
\hline Medios extranjeros & 1 & 0,1 & 3 & 0,2 \\
\hline Otros medios nacionales & 1 & 0,1 & 0 & 0 \\
\hline TOTAL & 16 & $1,4 \%$ & 29 & $2,1 \%$ \\
\hline
\end{tabular}

Los bajos niveles de enlaces externos contabilizados, con todo, manifestaban la cautela de estas dos cabeceras para vehicular al usuario a informaciones ubicadas en otros portales. También era un rasgo de un fuerte localismo informativo, en concreto debido al tema elegido ${ }^{11}$.

\section{CONCLUSIONES}

Los dos diarios digitales analizados, muy representativos del panorama ciberperiodístico colombiano, mostraron una baja implementación de enlaces hipertextuales. En concreto, ELTIEMPO.com, líder del país según los datos consultados en Alexa.com, prácticamente tendía a excluir la inclusión de estas estrategias cuando almacenaba los contenidos alusivos al presidente Santos. Esta circunstancia se explica porque de un lado el mercado de medios colombiano se transformó espectacularmente en un breve periodo. Si en 2009 había un 33\% de usuarios que consumía algún tipo de cibermedio (por un 35\% de usuarios de prensa convencional), en apenas dos años los usuarios de Internet aumentaron hasta el 43\%, en tanto que los de la prensa en papel se estancaron en un 35\% (según datos consultados en EGM - Colombia, 2009; 2011). Es decir: en un plazo de dos años los cibermedios pasaron de estar por debajo en incidencia con respecto de los periódicos impresos, hasta auparse ocho puntos porcentuales por encima de sus semejantes en papel. 
Ese crecimiento del consumo de los cibermedios se veía acompañado por unas estrategias enclavadas en el lenguaje anterior: el marco conceptual del periodismo impreso todavía marcaba las pautas, en el año 2010, sobre la narración digital.

El tipo de almacenaje que hemos mostrado en los resultados de este trabajo señalaba dos preferencias compositivas generales:

a) En el caso de ELTIEMPO.com, asistimos a una supresión de los enlaces, con el fin de conservar la información sin las inferencias de la escritura hipertextual. Es este un proceso que podría enunciarse como la formolización, en tanto que se preserva el texto, aunque se eliminan esas partes susceptibles de causar errores ${ }^{12}$. El periodismo digital: "[...] es mucho más que trasladar contenidos de la prensa escrita a la Red; implica múltiples voces, abundantes posibilidades de presentar la información, de contextualizarla y de complementarla” (Sánchez, 2005, p. 49).

Sin embargo, algunos cibermedios prefieren formolizar sus contenidos para facilitar la adaptación de sus usuarios a la nueva escritura multipolarizada hipertextual.

b) En el caso de ELESPECTADOR.com, comprobamos tanto el mantenimiento de la escritura hipertextual, como la intensificación de ese tipo de escritura mediante la instalación de máquinas agregadoras automáticas. En la figura 3, por ejemplo, hemos enseñado un contenido rotulado el 12 de noviembre de 2010; en esa misma captura, en la parte derecha, aparece una caja de enlaces que redireccionan a contenidos publicados el mismo día de la consulta. Es este un proceso que podría enunciarse como la vivificación de la información, en tanto que se preservan los elementos textuales e hipertextuales e incluso estos últimos se refuerzan con el fin de conseguir acentuar la experiencia del usuario.

Figura 6. Dos formas de archivar el hipertexto: formolización vs vivificación

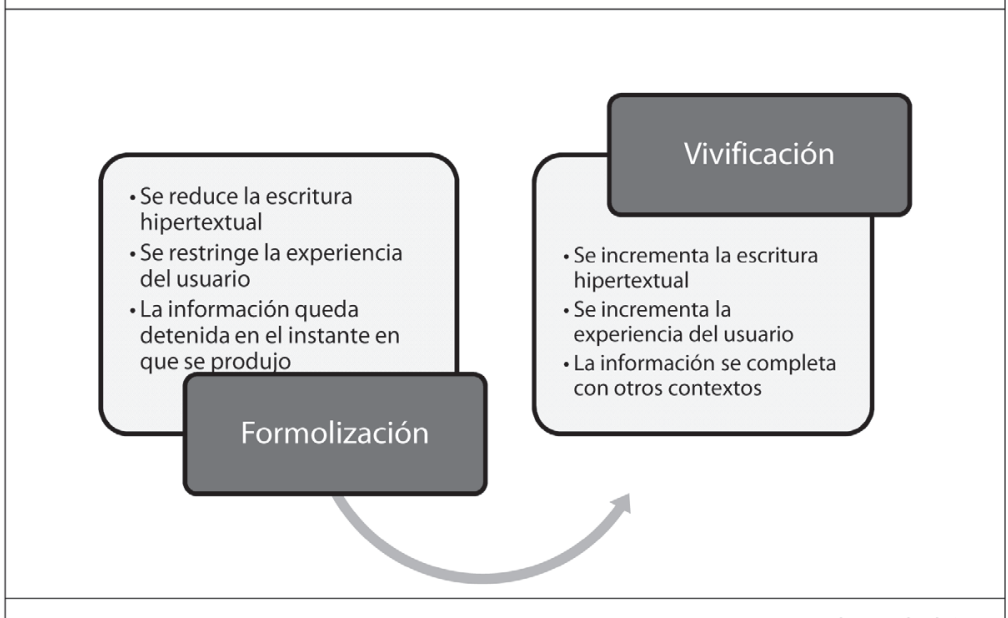

Fuente: Daniel Barredo lbáñez 
Tanto la formolización como la vivificación son procedimientos hegemónicos de almacenaje: el cibermedio ha de optar por uno u otro. Ambos ofrecen unas ventajas distintivas, de las cuales depende su inclusión.

Futuros estudios tendrían que examinar la evolución de la implementación hipertextual sobre las informaciones presidenciales o, en general, sobre otras informaciones simbólicamente representativas del quehacer del cibeperiodismo colombiano.

Anexo 1. ficha de análisis

\begin{tabular}{|c|c|}
\hline Identificación de la unidad & Enlaces internos \\
\hline V1. Fecha & $\begin{array}{l}\text { V9. ¿Hay enlaces a alguna noticia del día? } \\
0 \text {. No }\end{array}$ \\
\hline \multirow{2}{*}{ V2. Número de referencia } & 1. Sí \\
\hline & V10. ¿ Hay enlaces a noticias publicadas 3 días antes 0 \\
\hline V3. Mes de publicación & después? \\
\hline 8. Agosto & 0. No \\
\hline 9. Septiembre & 1. Sí \\
\hline 10. Octubre & V11. ¿Hay enlaces a noticias publicadas 4 días antes 0 \\
\hline 11. Noviembre & más? \\
\hline \multirow{2}{*}{ 12. Diciembre } & 0. No \\
\hline & 1. Sí \\
\hline Enlaces & V12. ¿Hay enlaces a noticias publicadas 4 días después 0 \\
\hline V4. ¿Cuántos enlaces hay? & más? \\
\hline 0. Ninguno & 0. No \\
\hline $1.1-2$ & 1. Sí \\
\hline $2.3-4$ & V13. ¿Hay enlaces a blogs del medio? \\
\hline $3.5-6$ & 0. No \\
\hline $4.7-8$ & 1. Sí \\
\hline $5.9-10$ & V14. ¿Hay enlaces a vídeos? \\
\hline \multirow[t]{3}{*}{ 6. 11 o más } & 0. No \\
\hline & 1. Sí \\
\hline & V15. ¿Hay enlaces a fotografías? \\
\hline Distribución & 0. No \\
\hline V5. ¿Los enlaces anteceden al texto? & 1. Sí \\
\hline 0. No & V16. ¿Hay enlaces a archivos de sonido? \\
\hline 1.Sí & 0. No \\
\hline V6. ¿Los enlaces forman parte del texto? & 1. Sí \\
\hline 0. No & V17. ¿Hay enlaces a infografías? \\
\hline 1. Sí & 0. No \\
\hline V7. ¿Los enlaces están en los laterales del texto? & 1. Sí \\
\hline \multicolumn{2}{|l|}{0. No } \\
\hline 1. Sí & \\
\hline V8. ¿Los enlaces están a continuación del texto? & Enlaces externos \\
\hline 0. No & V18. ¿Hay enlaces a otros medios del grupo? \\
\hline \multirow[t]{20}{*}{ 1. Sí } & 0. No \\
\hline & 1. Sí \\
\hline & V19. ¿Hay enlaces a otros medios nacionales? \\
\hline & 0. No \\
\hline & 1. Sí \\
\hline & V20. ¿Hay enlaces a medios extranjeros? \\
\hline & 0. No \\
\hline & 1. Sí \\
\hline & V21. ¿Hay enlaces a blogs externos? \\
\hline & 0. No \\
\hline & 1. Sí \\
\hline & V22. ¿Hay enlaces a documentos originales? \\
\hline & 0. No \\
\hline & 1. Sí \\
\hline & V23. ¿Hay enlaces a las fuentes? \\
\hline & 0. No \\
\hline & 1. Sí \\
\hline & V24. ¿ Hay enlaces a las redes sociales? \\
\hline & 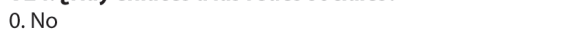 \\
\hline & 1. Sí \\
\hline
\end{tabular}


Anexo 2. Descripción de los enlaces internos

\begin{tabular}{|c|l|}
\hline Tipos & \multicolumn{1}{c|}{ Descripción } \\
\hline $\begin{array}{c}\text { Archivo } \\
\text { futuro }\end{array}$ & $\begin{array}{l}\text { Remiten a noticias del propio medio publicadas cuatro días después } \\
\text { del día de publicación de la unidad }\end{array}$ \\
\hline $\begin{array}{c}\text { Archivo } \\
\text { pasado }\end{array}$ & $\begin{array}{l}\text { Remiten a noticias del propio medio publicadas cuatro días antes del } \\
\text { día de publicación de la unidad }\end{array}$ \\
\hline Blog & Remiten a blogs alojados dentro del portal informativo \\
\hline $\begin{array}{c}\text { Fichero de } \\
\text { audio }\end{array}$ & $\begin{array}{l}\text { Identifican a esos recursos de sonido alojados dentro del portal } \\
\text { informativo }\end{array}$ \\
\hline $\begin{array}{c}\text { Fichero de } \\
\text { vídeo }\end{array}$ & $\begin{array}{l}\text { Identifican a esos recursos videográficos alojados dentro del portal } \\
\text { informativo }\end{array}$ \\
\hline Fotografía & $\begin{array}{l}\text { Identifican a esos recursos gráficos alojados dentro del portal } \\
\text { informativo }\end{array}$ \\
\hline Infografía & $\begin{array}{l}\text { Identifican a esos recursos infográficos alojados dentro del portal } \\
\text { informativo }\end{array}$ \\
\hline Noticia del & $\begin{array}{l}\text { Remiten a noticias del propio medio publicadas el mismo día de } \\
\text { publicación de la unidad analizada }\end{array}$ \\
\hline Otra noticia & $\begin{array}{l}\text { Remiten a noticias del propio medio publicadas tres días antes o tres } \\
\text { días después del día de publicación de la unidad }\end{array}$ \\
\hline & Fuente: Daniel Barredo lbáñez \\
\hline
\end{tabular}

\section{Notas}

${ }^{1}$ Este trabajo ha contado con una ayuda de $1400 €$ de la Consejería de Innovación, Ciencia y Empresa de la Junta de Andalucía como patrocinador del Programa de Becas de Movilidad Académica entre universidades andaluzas y latinoamericanas asociadas a la AUIP (Convocatoria 2012), en el marco de una estancia realizada entre el 18/01/2013 y el 18/05/2013 en la Universidad de La Sabana (Colombia); asimismo, el proceso de su redacción fue financiado por el Proyecto Prometeo de la Secretaría de Educación Superior, Ciencia, Tecnología e Innovación de la República del Ecuador, así como a través de los fondos propios de los autores.

${ }^{2}$ Suele explicarse el nacimiento del hipertexto a partir de las palabras de Vannevar Bush recogidas en 1945 en The Atlantic Monday (en Madrid López, 2010, p. 23).

${ }^{3}$ La ficha se entrega como Anexo 1 de este trabajo.

${ }^{4}$ Según Alexa.com, datos consultados el 20/04/2013 de: http://www.alexa.com/siteinfo/eltiempo.com

${ }^{5}$ Según Alexa.com, datos consultados el 20/04/2013 de: http://www.alexa.com/siteinfo/elespectador.com

${ }^{6}$ Las siguientes capturas han sido tomadas el 30/04/2013 de: http://www.elespectador.com/impreso/articuloimpreso234679-fondelibertad-intervenido

${ }^{7}$ El trabajo citado no estudiaba específicamente un fenómeno, como este estudio, sino que analizaba enlaces integrados en una amplia variedad temática.

${ }^{8}$ Fondevila y Segura (2012b) explican en su observación que ELTIEMPO.com introducía una media de 0,51 enlaces por unidad.

${ }^{9}$ Son esos enlaces que remiten al usuario a contenido emplazados en la propia cabecera. En el anexo 2 se adjunta una descripción pormenorizada de las características de los ítems aludidos en la Tabla 3.

${ }^{10}$ Son esos enlaces que remiten al usuario a contenidos emplazados fuera de la cabecera. En el anexo 3 se adjunta una descripción pormenorizada de las características de los ítems aludidos en la Tabla 4.

${ }^{11}$ Los índices de remisión a contenidos externos fluctúan en función de los temas; un análisis sobre el presidente de Estados Unidos, por ejemplo, puede que contenga más referencias a otros cibermedios que un análisis sobre el presidente de Colombia.

${ }^{12}$ Nos referimos en concreto a errores técnicos, como por ejemplo la remisión a un contenido que ha cambiado de dirección física. Es este un error muy común en las hemerotecas de los ciberdiarios de orientación general, como hemos comprobado en estudios complementarios a este (Barredo y Oller, 2012a; 2012b). 


\section{Referencias}

Armentia, J. I., Caminos, J. M., Elexgaray, J., Marín, F. \& Merchán, I. (2000). El Diario Digital.Análisis de los contenidos textuales, aspectos formales y publicitarios. Barcelona: Bosch.

Barredo, D. (2013). Fuentes de información y enlaces hipertextuales en las alusiones al rey Juan Carlos en ELPAÍS.com y ABC. es (2009-2011). Comunicación y Hombre, 9(12), p. 89 - 115.

Barredo, D. \& Oller, M. (2012a). Género, ideología y prensa digital. La construcción informativa del Día Internacional de la Mujer Trabajadora en www.abc.es y www.elpais.com (2001 - 2010). Revista Mediterránea de Comunicación: Alicante. Consultado el 12/05/2013 de: http://www.rmedcom.org/libros/2Barreda-Oller.pdf

Barredo, D. y Oller, M. (2012b). Las fuentes de información y los enlaces hipertextuales en las coberturas informativas de los enfrentamientos entre Real Madrid C. F. y F. C. Barcelona en MARCA.com y SPORT.es (2010-11). Miguel Hernández Journal of Communication, 3. Consultado el 12/05/2013 de: http://mhcj.es/2012/11/30/barredo_oller/

Cea D’ancona, M. Á. (1996). Metodología cuantitativa. Estrategias y técnicas de investigación social. Madrid: Síntesis.

Deuze, M. (2001). Online journalism: Modelling the first generation of news media on the World Wide Web. First Monday, 6(10). Consultado el 19/10/2012 de: http://www.firstmonday.org/htbin/cgiwrap/bin/ojs/index.php/fm/article/ view/893/802.

Díaz Noci, J., Codina, LL., Da Fonseca, L. et al. (2009). Content and message analysis of online journalism: some methodological proposals. Trípodos (Extra 2009). V Congrés Internacional. Comunicació y realitat, 647 - 656. Barcelona, Facultad de Comunicación Blanquerna.

EGM - Colombia. (2009). Estudio General de Medios, nº 11. www.acimcolombia.com. Consultado el 28/07/2013 de: http://www.acimcolombia.com/archivos/FILE_DOC_PUBLICACION/CIFRAS\%20EGM\%20_\%20ABRIL\%20 2010.pdf

EGM - Colombia. (2011). Estudio General de Medios, n 23. www.acimcolombia.com. Consultado el 28/07/2013 de: http://www.acimcolombia.com/archivos/FILE_DOC_PUBLICACION/CIFRAS\%20EGM\%20-\%20ABRIL\%20 2010.pdf

Fondevila, J. F. \& Segura, H. (2012a). Uso del hipertexto en el ciberperiodismo: el caso de Colombia. Chasqui, Revista Latinoamericana de Comunicación, 117, p. 80 - 84.

Fondevila, J. F. y Segura, H. (2012b). Hipertextuality in digital journalism in Colombia. Hipertext.net, (10). Consultado el 09/08/2013 de:

http: / /www.upf.edu/hipertextnet/en/numero-10/hypertextuality-in-digital-journalism-in-colombia.html

Larrondo, A. (2009). La metamorfosis del reportaje en el ciberperiodismo: concepto y caracterización de un nuevo modelo narrativo. Comunicación y Sociedad, XXII(2), p. 59 - 88.

Larrondo, A. (2010). Propuesta metodológica para una aproximación empírica a los géneros ciberperiodísticos. Zer: Revista de estudios de comunicación, 15(28), p. $157-174$.

Llano, S. (2005). Hipermedia y interactividad en el periodismo digital colombiano. Palabra Clave, 12, p. 113 - 129.

Madrid López, R. I. (2010). Hacia un modelo de comprensión de hipertexto: el papel de las estrategias de lectura y la carga cognitiva <tesis doctoral>. Granada: Editorial de la Universidad de Granada.

Neuendorf, K. A. (2002). The content analysis guidebook. Thousand Oaks, CA: Sage.

Pérez Marco, S. (2003). El concepto de hipertexto en el periodismo digital. Análisis de la aplicación del hipertexto en la estructuración de las noticias digitales de tres periódicos españoles (www.elpais.es, www.elmundo.es, www.abc.es) $<$ tesis doctoral> . Madrid: Universidad Complutense.

Rubio Lacoba, M. \& Blanco García, J. C. (2010). Mejor que un buscador, un encontrador. Documentación de las Ciencias de la Información, 33, p. 273 - 287.

Sánchez, D. A. (2008). Estudio comparativo entre las versiones impresa y digital de los principales diarios colombianos. Revista Lasallista de Investigación, 5(2), p. 48 - 62.

Waniek, J. (2012). How information organisation affects users' representation of hypertext structure and content. Behaviour \& Information Technology, 31(2), p. $143-154$. 